\title{
San Giuseppe il modello della partecipazione nell'Eucaristia
}

San Giovanni Paolo II nell'enciclica Ecclesia de Eucharistia l'ultimo capitolo lo dedica a Maria e la chiama "Donna eucaristica". Volendo comprendere la profondità del mistero dell'Eucaristia bisogna andare alla "scuola di Maria". In questo modo si può riscoprire il legame stretto che esiste tra la Chiesa e l'Eucaristia, e poi imparare dalla Madre del Signore a vivere la vita dell'Eucaristia. Lei, come nessun altro, è legata e conduce i fedeli al Santissimo Sacramento. Non entrando nelle questioni della presenza di Maria nel Cenacolo, dove è stata istituita l'Eucaristia, si può avere la certezza che dall'inizio con tutta la Chiesa era assidua nell'insegnamento degli Apostoli e partecipava allo spezzare il pane (vedi At 2,42-47). Però Maria diventò la "Donna eucaristica" innanzi tutto attraverso il suo atteggiamento interiore. Bisogna accentuare la sua fede e la fiducia senza

\footnotetext{
${ }^{1}$ Prof. Universitá Adamo Mickiewicz di Poznań lib. doc. don Dariusz Kwiatkowski (29 ottobre 1963) sacerdote della diocesi di Kalisz. Ha svolto il corso di laurea presso il Pontificio Ateneo S. Anselmo, dove nel 1996 si sono laureato in liturgia con la tesi intitolata Gli effetti del sacramento degli infermi secondo nuovo Ordo Unctionis Infirmorum. Presso Pontificia Università San Tommaso d'Aquino di Roma., con la tesi intitolata Gli effetti del sacramento degli infermi secondo l'Ordo Unctionis Infirmorum e il Catechismo della Chiesa Cattolica. In riferimento alle fonti storiche e liturgiche, ho ottenuto il titolo di dottore in data 27 aprile 1998. Dal novembre 2001 é professore associato presso la Facoltà di Teologia dell'Università Adamo Mickiewicz di Poznań. Auttore di 13 monografie, di cui due tomi di Materiali per gli esercizi destinati agli studenti di liturgia. Oltre a ciò, ha pubblicato circa 80 articoli scientifici su importanti riviste teologiche polacche con l'assegnazione di un punteggio. Ha partecipato all'organizzazione dei Simposi di Giuseppologia e del X Congresso Internazionale di Giuseppologia, tenutosi a Kalisz nel 2009. Ha partecipato a circa 70 simposi scientifici (quindici dei quali ha visto nel ruolo di organizzatore). Nel corso dei simposi ha presentato circa 30 relazioni, due delle quali in lingua italiana (in occasione del Congresso Internazionale di Giuseppologia tenutosi in Messico nel 2013 e in Francia nel 2017); e-mail: kwiatkowski@post.pl. ORCID: 0000-0002-8014-0016.
} 
limiti nella Parola di Dio: accogliere nel suo seno e nascere il Salvatore del mondo, ed accompagnare Gesù fino alla sua morte, risurrezione ed ascensione. Maria canta il suo Magnificat nella prospettiva eucaristica. Ella loda il Padre per Gesù, in Gesù e con Gesù. Lo loda in tutto, non omettendo il sacrificio che attraverso il suo fiat incessantemente offriva a Dio. Il Papa sottolinea che è il vero e proprio atteggiamento eucaristico ${ }^{2}$.

Bisogna notare che gli ultimi due secoli hanno aumentato l'interesse per la persona di san Giuseppe, Protettore del Salvatore e Marito vergine di Santissima Vergine Maria. In onore di san Giuseppe sono sorti nuove celebrazioni, preghiere e canti. I teologi hanno riflettuto sul ruolo di san Giuseppe nella storia della salvezza e nella Chiesa. I papi hanno rilasciato documenti di diverso tipo, in cui lodavano le virtù del Patriarca di Nazareth, hanno invocato la sua intercessione e gli hanno affidato la cura di tutta la Chiesa. Nella teologia hanno cominciato sempre più a vedere la persona di san Giuseppe al fianco di Maria e Gesù. Hanno sottolineato che queste tre persone costituiscono una unità indissolubile e i loro ruoli, affidati loro da Dio nella storia della salvezza, si possono comprendere solo allora, quando li si vedono insieme. Cristologia, mariologia e giuseppologia per essere comprese in modo proprio, devono essere tra loro legate strettamente ed intrecciarsi vicendevolmente.

Giuseppe fu scelto da Dio per essere testimone e partecipe del mistero dell'Incarnazione. La sua missione è legata strettamente alla missione di Maria e Gesù. Ne s'esprime nel matrimonio virginale con Maria e nella paternità terrestre di Gesù. Non è possibile comprendere Gesù senza Maria, ma anche senza Giuseppe. Non è possibile comprendere Maria senza Gesù, ma anche senza Giuseppe. E non è possibile comprendere Giuseppe, se non lo vediamo come il padre protettore della Santa Famiglia ${ }^{3}$. Nella predica durante l'insediamento del nuovo custode della basilica di san Giuseppe a Kalisz, il vescovo Stanisław Napierała sottolineava:

Prima dobbiamo notare che nella storia della salvezza ci sono questi Tre - Gesù, Maria e Giuseppe - costituiscono la Santa Famiglia. Non è possibile compren-

\footnotetext{
${ }^{2}$ Cfr. Giovanni Paolo II, Enciclica Ecclesia de Eucharistia, Poznań 2003, 53-58.

${ }^{3}$ Vedi S. Napierała, Homilia wygloszona w uroczystość św. Józefa Oblubieńca NMP w bazylice św. Józefa w Kaliszu, 19 marca 2001 roku (Omelia pronunciata nella solennità di san Giuseppe Sposo BMV nella basilica di san Giuseppe a Kalisz, 19 marzo 2001), Pismo Kurii Diecezjalnej "Diecezja Kaliska" 35 (2001), p. 175-179 (d'ora in poi: DK); cfr. W. Seremak, Św. Józef - szczególny nauczyciel uczestnictwa w mesjańskiej misji Chrystusa (San Giuseppe - il maestro speciale della partecipazione nella missione mesianica di Cristo) [in:] Duchowość św. Józefa z Nazaretu (Spiritualità di san Giuseppe da Nazareth), red. M. Chmielewski, Lublin 2003, p. 59-72; T. Stramare, Tajemnica życia ukrytego Jezusa w centrum teologii "Redemptoris Custos" (Mistero della vita nascosta di Gesù nel centro della teologia di "Redemptoris Custos"), "Kaliskie Studia Teologiczne" 4 (2005), p. 35-48 (d'ora in poi: KST).
} 
dere nessuno di Loro senza unione con gli altri due. Non è possibile comprendere Gesù senza Maria, sua Madre, ma anche senza Giuseppe. Non è possibile comprendere Maria senza Gesù, ma anche senza Giuseppe. Naturalmente non è possibile comprendere san Giuseppe senza Gesù e Maria. San Giuseppe insieme con Maria ha ricevuto la rivelazione sull'incarnazione del Figlio di Dio. Prima l'ha ricevuto Maria. Ha saputo che deve essere la Madre del Figlio di Dio e che lo si compirà con l'opera di Spirito Santo. Ha saputo che sarà Madre e Vergine. Dopo ebbe la sua annunciazione San Giuseppe. Ha saputo di Maria che è la Madre del Figlio di Dio e la Vergine. Ha saputo anche che è la volontà di Dio che prendesse Maria da se e La custodisse. In questo modo san Giuseppe, che era vergine diventò il padre legale di Gesù 4 .

Dio ha consegnato a san Giuseppe i più grandi tesori: suo Figlio e la Sua Madre. Proprio qui c'è la grandezza di san Giuseppe. La partecipazione di san Giuseppe nel piano della salvezza di Dio risulta dal suo matrimonio con Maria, Madre di Dio e dal mandato che Giuseppe ha ricevuto da Dio riguardo Gesù Salvatore e la Sua Madres.

Considerando le relazioni di san Giuseppe con Gesù e Maria, la sua missione nella storia della salvezza e nella storia di Chiesa, di cui è patrono e custode, attraverso il suo atteggiamento interiore e le attività intraprese si può chiamare san Giuseppe "il modello della partecipazione nella Eucaristia". Evidentemente vi è una certa analogia a chiamare Maria "Donna eucaristica" e non possiamo mettere in tutto il segno d'uguaglianza, però questo titolo spetta a san Giuseppe. Il suo atteggiamento interiore, che permette di definirlo così, si esprime nel fatto che lui era l'uomo di fede e d'amore, l'uomo della Parola di Dio, offrendo il sacrificio della sua vita, per compiere la volontà di Dio. In fine è il patrono della Chiesa che vive grazie all'Eucaristia.

\section{San Giuseppe l'uomo di fede e d'amore}

L'inserirsi di san Giuseppe nei piani della salvezza di Dio era possibile grazie alla sua fede. Giovanni Paolo II nell'esortazione Redemptoris Custos presenta san

${ }^{4}$ S. Napierała, Dar i skarb. Kazanie wygłoszone podczas wprowadzenia ks. kan. dr. J. Ploty $w$ urząd proboszcza parafii Wniebowzięcia NMP i kustosza sanktuarium św. Józefa w Kaliszu, 7 października 2001 roku (Dono e tesoro. Discorso pronunciato durante l'introduzione di don can. Dr J. Plota all 'ufficio di parroco della parrocchia d'Assunzione BMV e di custode del santuario di san Giuseppe a Kalisz, 7 ottobre 2001), DK 38 (2001), p. 98-101, qui: p. 99 (trad. D. Kwiatkowski).

${ }^{5}$ Cfr. J. Stöhr, Józef ,,sprawiedliwy”, dziewiczo zjednoczony węztem matżeńskim z Maryja (Giuseppe "giusto", verginalmente unito con il vincolo di matrimonio con Maria), KST 2 (2003), p. 49-53. 
Giuseppe come l'uomo di fede e d'obbedienza. Il Papa unisce la fede di Giuseppe alla fede di Maria ed annota: "All'inizio di questa peregrinazione la fede di Maria si incontra con la fede di Giuseppe" . Le parole di Elisabetta che riguardano la fede di Maria (vedi Lc 1,45) si possono riferire a san Giuseppe, perché positivamente e affermativamente ha risposto alla Parola di Dio con le opere concrete: "Dimostrò in tal modo una disponibilità di volontà, simile a quella di Maria, in ordine a ciò che Dio gli chiedeva per mezzo del suo messaggero"7. Ciò che ha fatto Giuseppe era la purissima obbedienza di fede. La fede e l'obbedienza nella vita di san Giuseppe si intrecciano e completano vicendevolmente ${ }^{8}$.

La via nella vita di san Giuseppe era la peregrinazione nella fede. Similmente come Maria, fino alla fine della sua vita è rimasto fedele alla chiamata di Dio. Maria il suo consenso per realizzare la volontà di Dio l'ha espresso attraverso il fiat detto a Gabriele durante l'annunciazione. Invece Giuseppe non proferì alcuna parola, ma "fece come gli aveva ordinato l'angelo del Signore" (Mt 1,24). Giovanni Paolo II afferma che "il silenzio di Giuseppe ha una speciale eloquenza: grazie ad esso si può leggere pienamente la verità contenuta nel giudizio che di lui dà il Vangelo: il «giusto» (cfr. Mt 1,19)"

San Giuseppe nell'annunciazione notturna ha scoperto di nuovo la sua vocazione e l'ha accettata, confermando le sue opere ulteriori. Giuseppe per sempre si era legato d'amore sponsale alla sua moglie Maria. Giovanni Paolo II scrive: "Quest'uomo «giusto» che, nello spirito delle più nobili tradizioni del popolo eletto, amava la Vergine di Nazareth ed a lei si era legato con amore sponsale, è nuovamente chiamato da Dio a questo amore" ${ }^{10}$.

L'amore sponsale di Giuseppe a Maria è il dono che viene dallo Spirito Santo. Il Papa osserva che Giuseppe era obbediente allo Spirito ed allora in Lui ha trovato la fonte d'amore sponsale maschile, alla sua Moglie verginale. Questa unione sponsale di Santi Sposi da Nazareth la esalta anche la liturgia della Chiesa. Nella prefazione del formulario della Messa della Santissima Maria Vergine da Nazareth, richiamando la vita familiare della Santa Famiglia a Nazareth, la Chiesa loda

\footnotetext{
${ }^{6}$ Giovanni Paolo II, Esortazione apostolica sulla figura e la missione di san Giuseppe nella vita di Cristo e della Chiesa, Redemptoris Custos, 15 agosto 1989, Roma 1989, 4 (d'ora in poi: RC).

7 Ibidem, 3.

${ }^{8}$ Cfr. W. Czamara, Glówne idee adhortacji apostolskiej Jana Pawła II „, Redemptoris Custos” (Idee principali d'Esortazione apostolica di Giovanni Paolo II "Redemptoris Custos"), "Ateneum Kapłańskie" 539 (1999), p. 6-12, qui: p. 9-10; J. Galot, Święty Józef (San Giuseppe), Kraków 1997, p. 73-78; T. Stramare, Syn Józefa z Nazaretu (Figlio di Giuseppe da Nazareth), Kraków 1997, p. 43-48.

${ }^{9}$ RC 17; cfr. M. Peter, „Mąż sprawiedliwy” (św. Józef wedtug tekstu Mateusza 1,19) ("Uomo giusto" [san Giuseppe secondo il testo di Matteo 1,19]), "W drodze" 7 (1979) 2, p. 48-56; K. Romaniuk, ,Józef, maż sprawiedliwy...” (Mt 1,19) ("Giuseppe, Uomo giusto...” [Mt 1,19]), “Collectanea Theologica" 50 (1980) 3, p. 25-34 (trad. D. Kwiatkowski).

${ }^{10} \mathrm{RC} 19$.
} 
Dio con le parole seguenti: "Vi Vergine purissima, unita con Giuseppe, l'uomo giusto, con il legame strettissimo d'amore verginale..." ${ }^{11}$. Giovanni Paolo II nella sua esortazione sottolinea il carattere particolare del vincolo sponsale di Giuseppe con Maria: "Mediante il sacrificio totale di sé Giuseppe esprime il suo generoso amore verso la Madre di Dio, facendole «dono sponsale di sé». Pur deciso a ritirarsi per non ostacolare il piano di Dio che si stava realizzando in lei, egli per espresso ordine angelico la trattiene con sé e ne rispetta l'esclusiva appartenenza a Dio" ${ }^{12}$.

Giovanni Paolo II nell'esortazione Redemptoris Custos mostra il Patriarca di Nazareth come l'uomo di profonda vita spirituale. Prima il Papa rivolge l'attenzione sul silenzio di Giuseppe, che in modo speciale rivela il profilo di questa figura. Il suo silenzio si distingueva nella profonda contemplazione del mistero di Dio, che dimorava in casa sua ${ }^{13}$. San Giuseppe era l'uomo che nella vita quotidiana riusciva conciliare l'azione con la contemplazione. Giuseppe era l'uomo di devozione profonda e vera. Il Papa afferma: „Questa sottomissione a Dio, che è prontezza di volontà nel dedicarsi alle cose che riguardano il suo servizio, non è altro che l'esercizio della devozione, la quale costituisce una delle espressioni della virtù della religione"14. La vicinanza a Gesù e il donarsi vicendevolmente hanno fatto di Giuseppe l'esempio luminoso della vita interiore ${ }^{15}$.

Anche il Papa Benedetto XVI nel suo insegnamento accentuava fortemente il posto e il ruolo di san Giuseppe nei piani salvifici di Dio. Nell'omelia pronunciata il 18 marzo 2009 durante i Vespri celebrati nella basilica di Maria Regina degli Apostoli a Jaunde, il Papa disse:

Giuseppe ha infatti vissuto alla luce del mistero dell'Incarnazione. Non solo con una prossimità fisica, ma anche con l'attenzione del cuore. Giuseppe ci svela il segreto di una umanità che vive alla presenza del mistero, aperta ad esso attraverso i dettagli più concreti dell'esistenza. In lui non c'è separazione tra fede e azione. La sua fede orienta in maniera decisiva le sue azioni. Paradossalmente è agendo, assumendo quindi le sue responsabilità, che egli si mette da parte per lasciare a Dio la libertà di realizzare la sua opera, senza frapporvi ostacolo ${ }^{16}$.

${ }^{11}$ Zbiór Mszy o Najświętszej Maryi Pannie (Raccolta delle Messe su Santissima Maria Vergine), Poznań 1998, p. 56 (trad. D. Kwiatkowski).

${ }^{12} \mathrm{RC} 20$.

${ }^{13}$ Cfr. ibidem 25.

${ }^{14}$ Ibidem, 26.

${ }^{15}$ Cfr. R.M. Kolinko, Św. Józef wzorem niezastapionej roli ojca $w$ świetle adhortacji apostolskiej Jana Pawła II „Redemptoris Custos” (San Giuseppe insostituibile esempio di ruolo del padre nell'Esortazione apostolica di Giovanni Paolo II "Redemptoris Custos"), KST 1 (2002), p. 354-355.

${ }^{16}$ Benedykt XVI, Nieszpory w Bazylice Maryi Królowej Apostołów, 18 marca 2009 r. Jaunde. Stuga musi być $i$ wierny, i roztropny (Vespri celebrati nella basilica di Maria Regina degli Apostoli, 
Questa vicinanza ed impegno di Giuseppe nei piani salvifici di Dio il Papa li ha accentuati in tante altre espressioni ${ }^{17}$.

L'Evangelista Matteo, descrivendo l'annunciazione di Giuseppe, ha sottolineato che lui era l'uomo giusto (cfr. Mt 1,19). Nel linguaggio biblico la giustizia è sinonimo di santità. Il Papa Benedetto XVI insegnava che la giustizia di Giuseppe risultava dal fatto che tutta la sua vita era subordinata alla volontà di Dio: "Giuseppe è «l'uomo giusto» (Mt 1,19), perché la sua vita è conformata alla Parola di Dio"18. Si può dire che Giuseppe in modo perfetto ha compiuto il mandato di Gesù affidato a tutti i Suoi discepoli: "Cercate invece, anzitutto, il regno di Dio e la sua giustizia, e tutte queste cose vi saranno date in aggiunta" (Mt 6,33). Non c'è dubbio che tutta la vita di Santa Famiglia era subordinata alle cose che appartengono al Padre (cfr. Lc 2,49 $)^{19}$. Nella sua dimensione umana Gesù grazie a Giuseppe ha conosciuto la via della santità e della giustizia. In questo contesto san Giuseppe diventa l'esempio di responsabilità per il dono della chiamata alla vita, la cui caratteristica essenziale è la giustizia evangelica, cioè la santità.

San Giuseppe è anche l'esempio d'amore. Prima bisogna vedere l'amore di Dio per san Giuseppe, e poi l'amore di Giuseppe per Dio e per gli uomini. La fonte e l'inizio della vocazione di Giuseppe come padre adottivo di Gesù e come lo Sposo di Maria era l'amore di Dio. Il Papa Benedetto XVI riguardo alla persona di san Giuseppe ha applicato lo stesso principio che permette di interpretare correttamente ogni vocazione che l'uomo riceve da Dio. Ogni vocazione ha la sua fonte nell'amore di $\mathrm{Dio}^{20}$. Solo nella prospettiva d'amore si può spiegare la grandezza della vocazione che san Giuseppe ha ricevuto da Dio. La sua vocazione era quasi inserita nella vocazione di Maria, perché Dio nel Suo amore ha destinato Maria per san Giuseppe e san Giuseppe per Maria, e loro due per Gesù̀ ${ }^{21}$. L'amore fa che Dio ha condiviso con san Giuseppe la paternità: "In realtà, uno solo è

18 marzo 2009, Jaunde. Servitore deve essere fedele e saggio), "L'Osservatore Romano", Città del Vaticano (ed. polacca) 5 (2009) 313, p. 26-30, qui: p. 28 (d'ora in poi: LO; trad. D. Kwiatkowski).

17 Vedi: idem, Aniol Pański 18 grudnia 2005 r. (Angelus, 18 dicembre 2005), LO 2 (2005) 280, p. 13; idem, Aniot Pański 19 marca 2006 r. (Angelus, 19 marzo 2006), LO 8 (2006) 285, p. 40; idem, Aniot Pański 31 grudnia 2006 r. (Angelus, 31 dicembre 2006), LO 2 (2007) 290, p. 51; idem, Anioł Pański 18 marca 2007 r. (Angelus, 18 marzo 2007), LO 5 (2007) 293, p. 55; idem, Aniot Pański 1 marca 2009 r. (Angelus, 1 marzo 2009), LO 5 (2009) 313, p. 56 e 58; idem, Audiencja generalna 20 grudnia 2006 r. (Udienza generale 20 dicembre 2006), LO 2 (2007) 290, p. 39.

${ }^{18}$ Idem, Nieszpory ..., p. 28.

${ }^{19}$ Cfr. idem, Odrzucajcie niszczycielska moc nienawiści, homilia wygłoszona w Nazarecie 14 maja 2009 roku (Rifiutate il potere distruttivo dell'odio. Omelia pronunciata a Nazaret, 14 maggio 2009), LO 7-8 (2009) 315, p. 40.

${ }^{20}$ Ibidem.

${ }^{21}$ Cfr. A. Latoń, Kierunki we współczesnej józefologii [in:] Duchowość św. Józefa z Nazaretu (Spiritualità di san Giuseppe da Nazareth), red. M. Chmielewski, Lublin 2003, p. 303-330, qui: p. 318. 
Padre - Dio Padre, unico Creatore di cose ed invisibili. Però all'uomo, creato a immagine e similitudine di Dio, è dato partecipare nella paternità particolare di Dio (cfr. Ef 3,15). In modo affascinante lo si esprime nella persona di san Giuseppe che è il padre senza la paternità carnale"22. San Giuseppe è stato scelto da Dio alla dignità di padre adottivo. In modo particolare Benedetto XVI ha "espresso questa verità nell'omelia pronunciata a Nazareth: Nella Santa Famiglia di Nazareth grazie a Gesù Maria e Giuseppe hanno conosciuto la grandezza dell'amore di Dio, Suo Padre Celeste, la sorgente definitiva d'ogni amore"23.

San Giuseppe ha risposto all'amore di Dio con l'amore per Dio e per l'uomo. Lui in modo perfetto ha fatto della sua vita un dono d'amore per Dio e per l'uomo. Nell'omelia pronunciata a Nazareth, durante il pellegrinaggio in Terra Santa, Benedetto XVI disse: "Nel falegname di Nazareth (Gesù) ha visto che l'autorità nel servizio d'amore porta i frutti immensamente più ricchi che l'autorità il cui lo scopo è la dominazione"24. L'amore di san Giuseppe in modo speciale era visibile nella relazione con Gesù e Maria. S'esprimeva nella sollecitudine senza limiti e nella custodia della Santa Famiglia. Lui durante tutta la sua vita ed attraverso tutte le sue opere con amore ed umiltà serviva Gesù e Maria. Perciò il Papa Benedetto XVI, ponendo Giuseppe come esempio dell'amore sponsale e paterno, frequentemente alla sua protezione affida i matrimoni e le famiglie, e chiama ad imitare il suo amore. Nello stesso tempo il Papa fa attenzione sull'autenticità dell'amore di Giuseppe per Gesù e Maria ${ }^{25}$.

Le riflessioni descritte sopra, che mostrano san Giuseppe come uomo di fede profonda e d'amore, fanno di lui in modo naturale l'Uomo eucaristico, benché mai ne partecipava. Ricevere l'Eucaristia e parteciparla esige dall'uomo la fede e l'amore. Solo la fede permette di riscoprire la presenza e l'azione di tutta la Santissima Trinità nel mondo dei segni liturgici. Anche nella fede si riscopre e si riceve l'opera salvifica attualizzata nell'Eucaristia da Cristo. La fede ci ordina di vedere sotto le specie del pane e del vino il vero Corpo e Sangue di Gesù Cristo (cfr. Gv 6,53-57) ${ }^{26}$. La partecipazione all'Eucaristia attraverso la fede trasforma la vita dell'uomo e la fa eucaristica, cioè totalmente sottomessa a compiere la volontà del Padre.

L'amore sponsale di Giuseppe per Gesù e Maria ancora di più lo avvicina all'Eucaristia che è l'attualizzazione dell'amore di Dio per l'umanità. L'amore di Giuseppe veniva dalla sua fede. L'amore ha fatto sì che abbia preso Maria come moglie e abbia riconosciuto Gesù come figlio suo. Lo ha accolto, affidandogli nel-

\footnotetext{
${ }^{22}$ Benedykt XVI, Nieszpory..., p. 26 (trad. D. Kwiatkowski).

${ }^{23}$ Idem, Odrzucajcie niszczycielska moc nienawiści..., p. 40 (trad. D. Kwiatkowski).

${ }^{24}$ Ibidem.

${ }^{25}$ Cfr. Benedykt XVI, Nieszpory..., p. 27-28.

${ }^{26}$ Cfr. CCC 1330-1344.
} 
lo stesso tempo se stesso. La relazione d'amore sponsale di Cristo per la Chiesa e della Chiesa per Cristo costituisce il fondamento dell'Eucaristia. Nell'Eucaristia Cristo si consegna totalmente alla Chiesa nell'atto d'amore disinteressato. San Giuseppe s'è consegnato a Dio e ha accolto i Suoi piani riguardo la sua persona anche nell'atto di amore totale per Dio.

\section{San Giuseppe l'uomo della Parola di Dio}

L'uomo eucaristico è l'uomo della Parola di Dio. Lui ascolta la Parola di Dio, la medita nel suo cuore e la vive. La liturgia della Parola di Dio costituisce la parte integrale d'ogni Eucaristia. La Costituzione liturgica, mostrando le forme della presenza di Cristo nella liturgia, ha rivolto l'attenzione sulla Sua presenza nella Parola di Dio ${ }^{27}$. Invece il Papa Benedetto XVI nell'Esortazione Verbum Domini dice direttamente l'aspetto sacramentale della Parola di Dio nell'Eucaristia. Benedetto XVI rivolge l'attenzione sulla presenza stabile di Cristo nella vita della Chiesa. Sottolinea che la relazione tra Cristo, che è il Verbo del Padre, e la comunità ecclesiale è straordinariamente vivace. Grazie a questa relazione la Parola di Dio è sempre presente nella comunità della Chiesa in virtù della promessa di Cristo: "Ed ecco, io sono con voi tutti i giorni, fino alla fine del mondo" (Mt 28,20). Nel contesto dell' Eucaristia il Papa parla dell'aspetto sacramentale della Parola di Dio e la prima sua fonte indica il mistero dell'Incarnazione: "il Verbo si fece carne" $(\mathrm{Gv} 1,14)$. È in questo momento che la Parola di Dio è entrata nella storia e nel tempo, rivelando nello stesso tempo il Padre e la pienezza della Rivelazione Divina ${ }^{28}$. L'aspetto sacramentale della Parola di Dio diventa comprensibile attraverso l'analogia con la presenza reale di Cristo sotto la specie del pane e del vino nell'Eucaristia. La Chiesa ha sempre creduto che "nel Santissimo Sacramento dell'Eucaristia è "contenuto veramente, realmente, sostanzialmente il Corpo e il Sangue di nostro Signore Gesù Cristo, con l'anima e la divinità e, quindi, il Cristo tutto intero"29. Nella Santa Comunione i credenti veramente ricevono il vero Corpo e Sangue di Cristo. La confessione di fede nella presenza di Cristo sotto la specie eucaristica porta con se anche la fede in ciò che Lui è presente e parla anche attraverso la Parola di Dio proclamata durante l'Eucaristia ${ }^{30}$.

${ }^{27}$ Cfr. SC 7.

${ }^{28}$ Cfr. Benedykt XVI, Adhortacja apostolska Verbum Domini (Esortazione apostolica Verbum Domini), Poznań 2010, 50-56.

${ }^{29}$ CCC 1374; cfr. Sobór Trydencki, Dekret o sakramencie Eucharystii (Concilio di Trento, Decreto sul sacramento d'Eucaristia) [in:] Breviarium fidei, red. S. Głowa, I. Bieda, Poznań 1988, 406-417, qui: 406, p. 203-208, qui: p. 204 (trad. D. Kwiatkowski); cfr. A. Szafrański, Teologia liturgii eucharystycznej (Teologia della liturgia eucaristica), Lublin 1981, p. 130-142.

${ }^{30}$ Cfr. Benedykt XVI, Adhortacja..., 56. 
San Giuseppe in tutto era obbediente a Dio ed aperto alla Sua Parola. In questa obbedienza educa Gesù e Lo prepara alla missione salvifica. Il Papa Paolo VI osserva che guardando la vita di san Giuseppe si può ancora meglio comprendere la Buona Notizia proclamata poi da Gesù Cristo. Il Vangelo delle "Beatitudini" cominciava nella casa familiare della Santa Famiglia a Nazareth. Paolo VI sottolineava che san Giuseppe diventò il tipo del Vangelo proclamato da Gesù ${ }^{31}$.

San Giuseppe è sempre un esempio stupendo non solo per gli stati di vita particolari ma anche per tutti i cristiani, mostrando chi sono e quali compiti hanno da svolgere nella loro vita terrena ${ }^{32}$. L'atteggiamento fondamentale d'ogni cristiano dovrebbe essere "devoto ascolto della Parola di Dio" 33 , cioè la disponibilità totale per compiere la volontà di Dio in tutto. In questo contesto il Papa osserva che "già all'inizio della Redenzione umana troviamo incarnato il modello dell'obbedienza, dopo Maria, proprio in Giuseppe, colui che si distingue per la fedele esecuzione dei comandi di Dio" 34 .

L'ascolto della Parola di Dio e la sua realizzazione mostrano gli episodi biblici con la partecipazione di san Giuseppe. È in ascolto ed obbediente alla Parola che Dio ha rivolto a lui. Questo atteggiamento ha espresso san Matteo con la corta affermazione: "Quando si destò dal sonno, Giuseppe fece come gli aveva ordinato l'angelo del Signore e prese con sé la sua sposa" (Mt 1,24). Queste parole senza equivoco indicano che san Giuseppe ha mostrato la fedeltà perfetta e l'obbedienza riguardo ai piani salvifici di Dio e la missione futura di Gesù. Lo stesso atteggiamento Giuseppe conservava per Maria, abbracciandola con il suo amore. La fedeltà di Giuseppe e la sua obbedienza alla volontà di Dio erano esposte a tante prove pesanti. Benedetto XVI sottolinea, che la prova speciale di fedeltà ed obbedienza fu la fuga in Egitto dal timore d'Erode e dei suoi piani per uccidere Gesù. San Giuseppe "per Cristo soffriva persecuzioni, esilio e indigenza. Doveva abbandonare la città familiare ed abitare lontano da essa" ${ }^{35}$. Questo episodio della vita della Santa Famiglia non si può distogliere dal contesto ampio della nascita di Gesù, perché praticamente tutte le circostanze unite erano per Giuseppe la verifica della sua fedeltà e obbedienza ai piani di Dio. Bisogna dire che da ognuna di queste prove Giuseppe usciva come l'uomo fedele e obbediente, e perciò lo possiamo chiamare l'esempio di fedeltà ed obbedienza provata.

San Giuseppe era l'uomo che ascoltava Dio, perché era l'esempio di preghiera e silenzio. Nella riflessione prima della preghiera dell' Angelus, il 18 dicembre 2005 Benedetto XVI ha rivolto l'attenzione su questo carattere di san Giuseppe e disse:

31 Paolo VI, S. Giuseppe, il modello degli umili, 19 marzo 1970 [in:] Insegnamenti di Paolo VI, Vol. 7 (1970), p. 1266-1269, qui: p. 1268.

32 Cfr. RC 29; W. Czamara, Gtówne idee..., p. 11.

$33 \mathrm{RC} 1$.

34 Ibidem 30.

35 Cfr. Benedykt XVI, Nieszpory..., p. 26 (trad. D. Kwiatkowski). 
Il suo è un silenzio permeato di contemplazione del mistero di Dio, in atteggiamento di totale disponibilità ai voleri divini. In altre parole, il silenzio di san Giuseppe non manifesta un vuoto interiore, ma, al contrario, la pienezza di fede che egli porta nel cuore, e che guida ogni suo pensiero ed ogni sua azione. Un silenzio grazie al quale Giuseppe, all'unisono con Maria, custodisce la Parola di Dio, conosciuta attraverso le Sacre Scritture, confrontandola continuamente con gli avvenimenti della vita di Gesù; un silenzio intessuto di preghiera costante, preghiera di benedizione del Signore, di adorazione della sua santa volontà e di affidamento senza riserve alla sua provvidenza ${ }^{36}$.

La preghiera vera e profonda in modo naturale si manifestava nel segreto e nel silenzio di Giuseppe. Perciò si può parlare di lui come di testimone silenzioso della contemplazione del mistero della Redenzione. Il Papa osservava anche che la pratica silenziosa della contemplazione quotidiana era d'esempio per Gesù. Il Salvatore del mondo, dal suo padre terrestre, guardandolo in modo umano, imparava la profonda vita interiore e la preghiera ${ }^{37}$.

San Giuseppe non solo ascoltava la Parola di Dio e la compiva ma lui ha accolto anche il Verbo Incarnato come figlio suo custodendolo come Padre naturale. Lui accompagnava il Verbo che ha preso il corpo umano dalla fanciullezza e Lo introduceva nella vita religiosa, culturale della sua nazione. Giuseppe viveva in grande unione con il Logos. Questo è lo scopo: proclamare ed ascoltare la Parola di Dio dai partecipanti all'Eucaristia. Essi devono ascoltare questa Parola ed identificarsi con essa. La Parola, che è lo stesso Gesù Cristo, desidera trasformare la vita umana e plasmarla sull'esempio della vita divina.

\section{San Giuseppe offrendo il sacrificio}

Il Catechismo della Chiesa Cattolica ha ricordato che: "Il nostro Salvatore nell'ultima Cena, la notte in cui veniva tradito, istituì il sacrificio eucaristico del suo Corpo e del suo Sangue, col quale perpetuare nei secoli, fino al suo ritorno, il sacrificio della croce, e per affidare così alla sua diletta Sposa, la Chiesa, il memoriale della sua Morte e Risurrezione"38. L'Eucaristia "porta indelebilmente inscritto l'evento della passione e della morte del Signore. Non ne è solo l'evocazione, ma la ri-presentazione sacramentale. È il sacrificio della Croce che si perpetua nei secoli" ${ }^{39}$. Perciò l'Eucaristia è il dono più prezioso nella comunità

\footnotetext{
36 Idem, Anioł Pański 18 grudnia..., p. 13 (trad. D. Kwiatkowski).

37 Ibidem.

38 CCC 1323; SC 47.

39 Giovanni Paolo II, Enciclica..., 11.
} 
della Chiesa. Qui Cristo offre se stesso e il dono della salvezza che non può essere limitato solo al futuro. La Chiesa, celebrando l'Eucaristia che è la memoria della morte e risurrezione di Cristo, rende questi avvenimenti realmente presenti. Giovanni Paolo II sottolineava che "Questo sacrificio è talmente decisivo per la salvezza del genere umano che Gesù Cristo l'ha compiuto ed è tornato al Padre soltanto dopo averci lasciato il mezzo per parteciparvi" ${ }^{40}$.

La vita terrena di san Giuseppe era segnata da lavoro pesante. Si può dire che tutta la sua vita costituiva il sacrificio di offerta di se stesso a Dio. Lo faceva servendo Gesù e Maria. Il Patriarca di Nazareth, così molto preoccupato per Gesù e Maria, esprimeva l'amore per loro attraverso il lavoro. Il suo lavoro assicurava alla Santa Famiglia le condizioni oneste per vivere. San Luca ha scritto nel suo Vangelo che dopo il ritorno da Gerusalemme, Gesù era obbediente ai suoi genitori (cfr. Lc 2,51). Questa sottomissione di Gesù si può intendere come la partecipazione al lavoro di Giuseppe. Giovanni Paolo II afferma: "Questa «sottomissione», cioè l'obbedienza di Gesù nella casa di Nazareth, viene intesa anche come partecipazione al lavoro di Giuseppe. Colui che era detto il «figlio del falegname» aveva imparato il lavoro dal suo «padre» putativo"41. Il lavoro umano, insieme con l'umanità del Figlio di Dio, era iscritto nel mistero dell'Incarnazione e Redenzione. Questo avvicinarsi del lavoro al mistero di Redenzione è avvenuto grazie a san Giuseppe che nel suo cantiere lavorava insieme con Gesù, il Figlio di Dio. La virtù di operosità di Giuseppe indica il ruolo importante del lavoro sia nella vita di famiglia come anche nella realtà della Redenzione ${ }^{42}$. In questo posto vale la pena annotare che nell'Eucaristia ci sono offerti i doni del pane e del vino, essendo i frutti del lavoro delle mani umane.

Le offerte compiute da san Giuseppe erano anche la sua castità ed onestà. Nel Vangelo di Matteo leggiamo: "Ecco, gli apparve in sogno un angelo del Signore e gli disse: «Giuseppe, figlio di Davide, non temere di prendere con te Maria, tua sposa. Infatti il bambino che è generato in lei viene dallo Spirito Santo» (Mt 1,20)". San Giuseppe era l'uomo onesto e perciò voleva uscire dalla sua vita per non offendere la sua reputazione. Proprio così bisogna intendere il progetto primordiale di Giuseppe sull'allontanare da se Maria. La decisione difficile di Giuseppe era il segno della sua castità. Il Papa Benedetto XVI accentuò molte volte che Maria era già sposata con Giuseppe, quando l'Angelo annunziò a Lei il messaggio sul concepimento e nascita di Gesù. San Giuseppe viveva nello splendore del mistero dell'Incarnazione: "Quando l'Angelo dell'annunciazione ha visitato Maria, Ella era già sposata con Giuseppe. Rivolgendosi direttamente a Maria, il Signore unisce strettamente Giuseppe con il mistero dell'Incarnazione.

\footnotetext{
40 Ibidem.

41 RC 22.

${ }^{42}$ Cfr. W. Czamara, Główne idee..., p. 10.
} 
Giuseppe ha accettato la partecipazione ai grandi eventi che Dio ha iniziato nel grembo della sua sposa. Ha preso Maria con $\mathrm{se}^{\text {"43. }}$. La sua decisione, nonostante tutti i dubbi precedenti ed incertezze, è il segno della sua integrità e castità. San Giuseppe come lo Sposo vergine di Maria diventò d'esempio per tutti i cristiani nell'osservare l'integrità e la castità nel contesto dei piani salvifici di Dio.

Custode del Salvatore e Sposo della Santissima Maria Vergine è anche l'esempio di sacrificio e prodezza. Questo atteggiamento mostra l'Evangelista Matteo: "Essi [Magi] erano appena partiti, quando un angelo del Signore apparve in sogno a Giuseppe e gli disse: «Àlzati, prendi con te il bambino e sua madre, fuggi in Egitto e resta là finché non ti avvertirò: Erode infatti vuole cercare il bambino per ucciderlo». Egli si alzò, nella notte, prese il bambino e sua madre e si rifugiò in Egitto" (Mt 2,13-14). San Giuseppe dimostra un atteggiamento di sacrificio e prodezza nelle circostanze del drammatico viaggio in Egitto. Prima lo mostrava, quando con la fiducia ha accolto Maria come sua moglie. In queste circostanze Giuseppe è per eccellenza il difensore della vita di Gesù, Salvatore del mondo. Perciò l'aiuto da san Giuseppe possono cercarlo tutti coloro che soffrono la persecuzione a causa della fede. Lui diventa per loro l'esempio di sacrificio e prodezza che penetra l'amore per Dio senza limiti ${ }^{44}$.

San Giuseppe dall'inizio avvolgeva Gesù e Maria di cura ed amore. Nella relazione con loro li travolge con grande bontà e premura: "Al vederlo restarono stupiti, e sua madre gli disse: «Figlio, perché ci hai fatto questo? Ecco, tuo padre e io, angosciati, ti cercavamo» (Lc 2,48)". Il perdere e ritrovare Gesù nel Tempio di Gerusalemme rivelano la premura paterna di san Giuseppe per Gesù. Benedetto XVI osserva che la risposta per la bontà di san Giuseppe era l'obbedienza di Gesù: "Nella vita trascorsa a Nazareth, Gesù ha onorato la Vergine Maria e il giusto Giuseppe, rimanendo sottomesso alla loro autorità per tutto il tempo della sua infanzia e adolescenza (cfr. Lc 2,51-52)" ${ }^{\prime 45}$. Essendo l'esempio di bontà e premura per la santa Famiglia, sulla tappa presente della storia di salvezza, san Giuseppe avvolge la Chiesa d'amore paterno ed implora i beni soprannaturali per tutti i suoi membri ${ }^{46}$.

Queste virtù di san Giuseppe si possono chiamare "virtù eucaristiche". Esse esprimono l'inserirsi nel sacrificio di Gesù Cristo attualizzato durante ogni Eucaristia. Tutti i fedeli sono chiamati ad offrire a Dio la loro vita insieme con Cristo e la Chiesa.

${ }^{43}$ Cfr. Benedykt XVI, Nieszpory..., p. 27.

${ }^{44}$ Cfr. Benedykt XVI, Afryka jest kontynentem nadziei (L'Africa é un continente di speranza. Omelia pronunciata a Jaunde), LO 5 (2009) 313, p. 30-32; idem, Nieszpory..., p. 27-28.

${ }^{45}$ Idem, Aniot Pański 31 grudnia ..., p. 51 (trad. D. Kwiatkowski).

${ }^{46}$ Cfr. idem, Afryka jest kontynentem nadziei..., p. 32. 


\section{Conclusione}

Le decisioni dei Papi Giovanni XXIII e Francesco e il documento della Congregazione per il Culto Divino e la Disciplina dei Sacramenti d'introdurre il nome di san Giuseppe nel Canone della Santa Messa, confermati prima dal Concilio Vaticano II, fatti in modo semplice, sono diventati la prova che il lungo dimenticare di san Giuseppe è finito definitivamente. Il suo nome è stato messo subito dopo Maria nella più importante e in una delle più antiche preghiere della Santa Messa. Bisogna sottolineare fortemente che dalla numerosa schiera di Apostoli e martiri solo il nome di Giuseppe s'è trovato nel Canone subito dopo la Santissima Vergine Maria. Questo significa che finalmente la Chiesa in modo ufficiale ha intravveduto il posto singolare del Patriarca di Nazareth nella storia della salvezza e nella storia della Chiesa. San Giuseppe in dignità e santità s'è trovato subito dopo Maria e in un certo modo è stato escluso dalla schiera degli altri santi per il suo legame particolare con Gesù e Maria.

Senz'altro introdurre il nome di san Giuseppe nelle preghiere eucaristiche della Chiesa in modo speciale permette di chiamarlo "l'Uomo eucaristico", nonostante che mai vi abbia partecipato in modo sacramentale. Però ne partecipa dall'inizio tra il numero immenso di celebranti della liturgia celeste (cfr. CCC 1137-1139). Ma prima di tutto il suo atteggiamento interiore e le attività intraprese gli permettono di essere chiamato "il modello della partecipazione nell'Eucaristia" e mostrare oggi ai fedeli che significa essere "l'uomo eucaristico" e in che cosa dovrebbe esprimersi la spiritualità eucaristica. Questo comportamento eucaristico si esprime nel fatto che san Giuseppe era l'uomo di fede e d'amore, l'uomo della Parola di Dio, offrendo il sacrificio della sua vita, per compiere la volontà di Dio.

\section{Saint Joseph as the example of participation in the Eucharist}

\section{Summary}

Pope John Paul II in the encyclical Ecclesia de Eucharistia called Mary a 'Woman of the Eucharist'. He pointed out the attitudes that can be described as Eucharistic. This article, using the principle of analogy and maintaining an appropriate balance, shows St. Joseph as a model of the Eucharistic approach of every Christian. The life of Saint Joseph was characterized by deep faith and love for God and man, the ability to hear and receive the word of God and the constant willingness to sacrifice his life in order to be able to fulfill the will of God. All these qualities are needed to participate in the Eucharist in a conscious and active way. These attitudes result from participation in the Eucharist and should shape the life of every Christian. In addition, it should be emphasized that the Church introduced the name of St. Joseph to the Eucharistic prayers and ordered it to be mentioned imme- 
diately after Mary. Placing the name of St. Joseph in the most important prayer of the Holy Mass, introduces him to the heart of the Eucharist.

\section{Keywords}

St. Joseph, Eucharist, faith, love, trust, God's word, service, sacrifice, God's will, model

\section{Święty Józef wzorem uczestnictwa w Eucharystii}

\section{Streszczenie}

Święty Jan Paweł II w encyklice Ecclesia de Eucharistia nazwał Maryję „Niewiastą Eucharystii”. Zwrócił uwagę na Jej postawy, które można określić jako eucharystyczne. Niniejszy artykuł, stosując zasadę analogii i zachowując odpowiednie proporcje, ukazuje św. Józefa jako wzór postaw eucharystycznych dla każdego chrześcijanina. Życie św. Józefa cechowała głęboka wiara i miłość do Boga oraz do człowieka, zdolność słuchania i przyjmowania słowa Bożego oraz nieustanna gotowość składania ofiary ze swojego życia, aby móc wypełnić wolę Bożą. Wszystkie te cechy są potrzebne, aby uczestniczyć w Eucharystii w sposób świadomy i czynny. Postawy te wynikają z uczestnictwa w Eucharystii i powinny kształtować życie każdego chrześcijanina. Poza tym należy podkreślić, że Kościół wprowadził imię św. Józefa do modlitw eucharystycznych i nakazał je wymieniać zaraz po Maryi. Umieszczajac imię św. Józefa w najważniejszej modlitwie mszalnej, wprowadził go w serce Eucharystii.

\section{Słowa kluczowe}

św. Józef, Eucharystia, wiara, miłość, ufność, słowo Boże, służba, ofiara, wola Boża, wzór
Abbreviazioni utilizzate
$\mathrm{CCC}$ - Catechismo della Chiesa Cattolica
DK - Pismo Kurii Diecezjalnej (Rivista della Curia Diocesana di Kalisz) "Diecezja Kaliska"
KST - Kaliskie Studia Teologiczne (Studi Teologici di Kalisz)
LO _ “L'Osservatore Romano", Città del Vaticano (ed. polacca)
RC - Esortazione apostolica Redemptoris Custos di Giovanni Paolo II
$\mathrm{SC} \quad$ - Concilio Vaticano II, La Costituzione liturgica Sacrosanctum Concilium.

\section{Bibliografia}

Benedykt XVI, Adhortacja apostolska Verbum Domini (Esortazione apostolica Verbum Domini), Poznań 2010. 
Benedykt XVI, Afryka jest kontynentem nadziei (L'Africa é un continente di speranza. Omelia pronunciata a Jaunde), LO 5 (2009) 313, p. 30-32.

Benedykt XVI, Aniot Pański 18 grudnia 2005 r. (Angelus, 18 dicembre 2005), LO 2 (2005) 280, p. 13.

Benedykt XVI, Anioł Pański 19 marca 2006 r. (Angelus, 19 marzo 2006), LO 8 (2006) 285, p. 40.

Benedykt XVI, Anioł Pański 31 grudnia 2006 r. (Angelus, 31 dicembre 2006), LO 2 (2007) 290, p. 51.

Benedykt XVI, Anioł Pański 18 marca 2007 r. (Angelus, 18 marzo 2007), LO 5 (2007) 293, p. 55.

Benedykt XVI, Anioł Pański 1 marca 2009 r. (Angelus, 1 marzo 2009), LO 5 (2009) 313, p. 56.

Benedykt XVI, Anioł Pański 15 marca 2009 r. (Angelus, 15 marzo 2009), LO 5 (2009) 313, p. 58.

Benedykt XVI, Audiencja generalna 20 grudnia $2006 r$. (Udienza generale 20 dicembre 2006), LO 2 (2007) 290, p. 39.

Benedykt XVI, Nieszpory w Bazylice Maryi Królowej Apostołów, 18 marca 2009 r. Jaunde. Stuga musi być $i$ wierny, i roztropny (Vespri celebrati nella basilica di Maria Regina degli Apostoli, 18 marzo 2009, Jaunde. Servitore deve essere fedele e saggio), LO 5 (2009) 313, p. 26-30.

Benedykt XVI, Odrzucajcie niszczycielska moc nienawiści. Homilia wygłoszona w Nazarecie 14 maja 2009 roku (Rifiutate il potere distruttivo dell'odio. Omelia pronunciata a Nazaret, 14 maggio 2009), LO 7-8 (2009) 315, p. 39-40.

Catechismo della Chiesa Cattolica. Testo integrale e commento teologico, Città del Vaticano 1994.

Czamara W., Główne idee adhortacji apostolskiej Jana Pawła II „Redemptoris Custos” (Idee principali d'Esortazione apostolica di Giovanni Paolo II "Redemptoris Custos"), “Ateneum Kapłańskie” 539 (1999), p. 6-12.

Galot J., Święty Józef (San Giuseppe), Kraków 1997.

Giovanni Paolo II, Enciclica Ecclesia de Eucharistia, Poznań 2003.

Giovanni Paolo II, Esortazione apostolica sulla figura e la missione di san Giuseppe nella vita di Cristo e della Chiesa, Redemptoris Custos, 15 agosto 1989, Roma 1989.

Kolinko R.M., Św. Józef wzorem niezastapionej roli ojca wświetle adhortacji apostolskiej Jana Pawła II „Redemptoris Custos” (San Giuseppe insostituibile esempio di ruolo del padre nell'Esortazione apostolica di Giovanni Paolo II "Redemptoris Custos", KST 1 (2002), p. 347-360.

Latoń A., Kierunki we współczesnej józefologii [in:] Duchowość św. Józefa z Nazaretu, (Spiritualità di san Giuseppe da Nazareth), red. M. Chmielewski, Lublin 2003, p. 303-330.

Napierała S., Dar i skarb. Kazanie wygłoszone podczas wprowadzenia ks. kan. dr. J. Ploty $w$ urząd proboszcza parafii Wniebowzięcia NMP i kustosza sanktuarium św. Jó- 
zefa w Kaliszu, 7 października 2001 roku (Dono e tesoro. Discorso pronunciato durante l'introduzione di don can. dr J. Plota all'ufficio di parroco della parrocchia d'Assunzione BMV e di custode del santuario di san Giuseppe a Kalisz, 7 ottobre 2001), DK 38 (2001), p. 98-101.

Napierała S., Homilia wygłoszona w uroczystość św. Józefa Oblubieńca NMP w bazylice św. Józefa w Kaliszu, 19 marca 2001 roku (Omelia pronunciata nella solennità di san Giuseppe Sposo BMV nella basilica di san Giuseppe a Kalisz, 19 marzo 2001), DK 35 (2001), p. 175-179.

Paolo VI, S. Giuseppe, il modello degli umili, 19 marzo 1970 [in:] Insegnamenti di Paolo VI, Vol. 7 (1970), p. 1266-1269.

Peter M., „Mąż sprawiedliwy” (św. Józef wedtug tekstu Mateusza 1,19) ("Uomo giusto” [san Giuseppe secondo il testo di Matteo 1,19]), "W drodze" 7 (1979) 2, p. 48-56.

Romaniuk K., „Józef, mąz sprawiedliwy...” (Mt 1,19) (“Giuseppe, Uomo giusto...” [Mt 1,19]), "Collectanea Theologica" 50 (1980), 3, p. 25-34.

Seremak W., Św. Józef - szczególny nauczyciel uczestnictwa w mesjańskiej misji Chrystusa (San Giuseppe - il maestro speciale della partecipazione nella missione mesianica di Cristo) [in:] Duchowość św. Józefa z Nazaretu (Spiritualità di san Giuseppe da Nazareth), red. M. Chmielewski, Lublin 2003, p. 59-72.

Sobór Trydencki, Dekret o sakramencie Eucharystii (Concilio di Trento, Decreto sul sacramento d'Eucaristia) [in:] Breviarium fidei, red. S. Głowa, I. Bieda, Poznań 1988, 406-417, p. 203-208.

Sobór Watykański II, Konstytucja o liturgii świętej Sacrosanctum Concilium (Concilio Vaticano II, La Costituzione liturgica Sacrosanctum Concilium) [in:] Sobór Watykański II, Konstytucje, dekrety, deklaracje (Concilio Vaticano II, Costituzioni, Decreti, Declarazioni), Poznań 2002, p. 48-78.

Stöhr J., Józef ,,sprawiedliwy”, dziewiczo zjednoczony węzłem małżeńskim z Maryja (Giuseppe "giusto", verginalmente unito con il vincolo di matrimonio con Maria), KST 2 (2003), p. 49-53.

Stramare T., Syn Józefa z Nazaretu (Figlio di Giuseppe da Nazareth), Kraków 1997.

Stramare T., Tajemnica życia ukrytego Jezusa w centrum teologii „Redemptoris Custos” (Mistero della vita nascosta di Gesù nel centro della teologia di "Redemptoris Custos"), KST 4 (2005), p. 35-48.

Szafrański A., Teologia liturgii eucharystycznej (Teologia della liturgia eucaristica), Lublin 1981.

Zbiór Mszy o Najświętszej Maryi Pannie (Raccolta delle Messe su Santissima Maria Vergine), Poznań 1998. 